\title{
Mandible Behavior in Obstructive Sleep Apnea Patients Under CPAP Treatment
}

\author{
F. Senny*, ${ }^{*}$, G. Maury ${ }^{2}$, L. Cambron ${ }^{3}$, A. Leroux ${ }^{1}$, J. Destiné ${ }^{1}$ and R. Poirrier ${ }^{3}$ \\ ${ }^{I}$ Electronic Department, Montefiore Insitute, B28, University of Liège (ULg), Liège (Sart-Tilman), Belgium \\ ${ }^{2}$ Pneumology Department, CHU Mont Godinne, YVOIR, Belgium \\ ${ }^{3}$ Neurology Department, Faculty of Medicine, B35, University of Liège (ULg), Liège (Sart-Tilman), Belgium
}

\begin{abstract}
Aim: To investigate whether obstructive sleep apnea (OSA) patients present different behaviors of mandible movements before and under CPAP therapy.

Materials and Methodology: In this retrospective study, patients were selected according to inclusion criteria: both the diagnostic polysomnography recording showing an OSA with an apnea-hypopnea index (AHI) greater than 25 (n/h) and the related CPAP therapy control recordings were available, presence of mandible movement and mask pressure signals in the recordings, and tolerance to the applied positive pressure. Statistical analysis on four parameters, namely the apneahypopnea index (AHI), the arousal index (ArI), the average of the mandible lowering during sleep (aLOW), and the average amplitude of the oscillations of the mandible movement signal (aAMPL), was performed on two sets of recordings: OSA and CPAP therapy.

Results: Thirty-four patients satisfied the inclusion criteria, thus both OSA and CPAP groups included thirty-four recordings each. Significant difference $(\mathrm{p}<0.001)$ was found in the OSA group compared with the CPAP group when considering either the four parameters or only the two ones related to mandible movements.

Conclusions: When an efficient CPAP pressure is applied, the mouth is less open and presents fewer broad sharp closure movements, and oscillating mandible movements are absent or very small.
\end{abstract}

Keywords: Mandible movement, sleep apnea, CPAP, statistical analysis.

\section{INTRODUCTION}

The inelegant though clear title "Shut your mouth and save your sleep" [1] points out the importance of nasal breathing over mouth breathing, thus the necessity to keep the mouth closed at night. Several authors showed relationships between sleep disordered-breathing events, respiratory effort and mouth opening, therefore making relevant the monitoring of the degree of mouth opening. Mouth opening increases upper airway collapsibility and thus the risk of occurrence of respiratory events [2]. Jaw motion was observed in case of blockage of the upper airway. Upon upper airway collapse, the pressure in the lungs becomes more and more subatmospheric and the jaw is passively lowered by thoracic attraction called thoracic tug [3]. The consequent opening of the mouth reflects, during sleep, a stronger effort to breathe. In [4-5], oscillating jaw movements (breathing frequency band [0.15-0.33] Hz) were observed in correlation with the esophageal pressure during obstructive sleep events. This correlation confirmed that the jaw movements could reflect the effort to breathe. In addition to presenting oscillations, the mandible lowers more during sleep in patients with OSA than in healthy subjects. Myamoto et al. used an intra-

*Address correspondence to this author at the Electronic Department, Montefiore Insitute, B28, University of Liège (ULg), Liège (Sart-Tilman), Belgium; Tel: +32 436626 80; Fax: +32 436629 50;

E-mail: F.Senny@ulg.ac.be oral magnetic sensor to measure mouth opening and they showed that OSA patients spent $70 \%$ of sleep time with a mandible opening greater than $5 \mathrm{~mm}$, compared with the results in a healthy population (11\% of sleep time). Significant mouth opening occurs during an obstructive apnea in NREM sleep, while in REM sleep, the muscle atonia attenuates these movements [6].

The purpose of this paper is to quantify the changes in the movements of the inferior jaw in an apneic population treated under CPAP, between the diagnostic PSG night and the CPAP treatment control night of the same patients. In the present study, the analysis of the mandible movement signal was performed by decomposing the average (degree of mouth opening) and oscillatory (amplitude of respiratory effort) components of the signal during sleep periods only.

\section{MATERIALS AND METHODOLOGY}

\section{a. Subjects and Recordings}

Diagnostic and CPAP control treatment PSG recordings (DPSG and CPSG) were selected retrospectively among the recordings made from January 2009 to December 2010 according to these inclusion criteria: (1) DPSG and CPSG recordings were paired, i.e. each pair came from the same patient ( 3 to 6 months between DPSG and CPSG), (2) mandible movement and mask pressure signals had been recorded in addition to the standard PSG signals, (3) the DPSG recording allowed to diagnose an OSA with an apnea- 

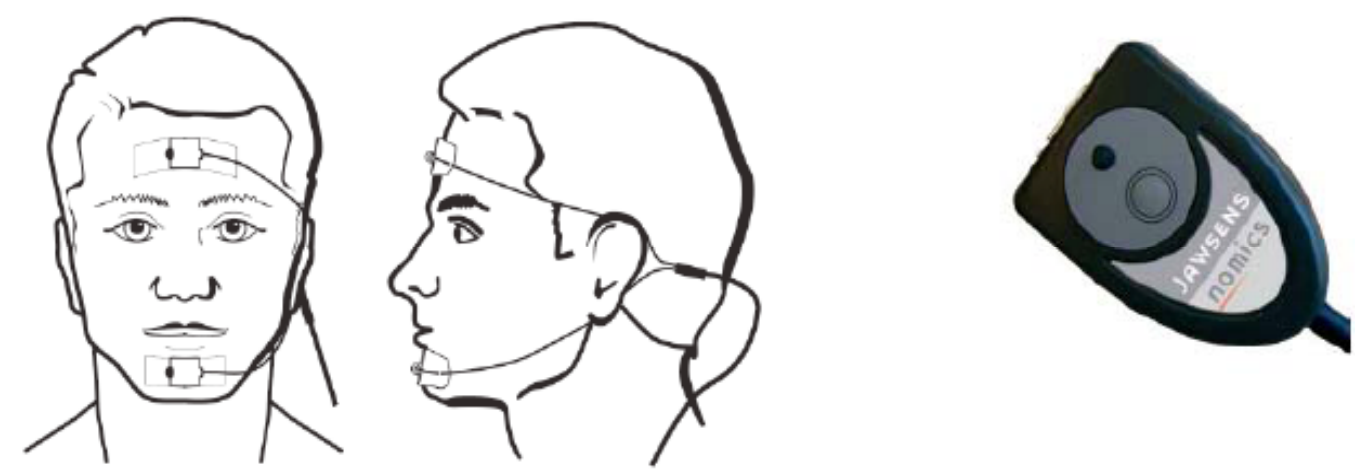

Fig. (1). Mandible movement sensor. The probes are placed on the median line of the face on the forehead and on the chin. The mandible movement signal corresponds to the distance between them. The Jawsens device that interfaces the sensor to the PSG system is illustrated on the right.

hypopnea index (AHI) greater than $25 \mathrm{n} / \mathrm{h}$, (4) the CPAP titration was performed previously manually on the basis of the Stradling equation pressure [7], and (5) the CPAP was applied through a nasal mask for at least four hours of sleep in CPSG. This study followed the principles of the Declaration of Helsinki, the patients were informed that the data could be used for research purpose and gave oral agreement.

The PSG recordings were made using a S7000 polysomnograph (EMBLA Medcare, Denver, US) and included the following neurophysiology signals: three-channel electroencephalography (EEG, C3-A2, C4-A1, FZ-CZ), left and right electrooculography (EOG), submental electromyography (chin EMG) for sleep staging and arousal scoring, two (left and right) tibial EMG for periodic leg movement evaluation. Also recorded were cardiorespiratory signals comprising ECG, nasal cannula/pressure transducer (NAF) (Protech, Mukilteo, US), chest and abdominal inductance plethysmography belts, a plethypulse, a blood oxygen oximeter $\left(\mathrm{SpO}_{2}\right.$, Nonin, Plymouth, US), a snoring sound detection (piezoelectric sensor from EMBLA), and a body position marker (body position sensor Protech).

\section{b. Mandible Movement Recording}

The movements of the mandible were acquired by a distance-meter based on the principle of magnetometry (Jawsens ${ }^{\circledR}$, NOMICS, Liège, Belgium). The sensor was composed of 2 probes, each probe containing a coil and a capacitor embedded in a small cylinder $(7 \mathrm{~mm}$ diameter; 25 $\mathrm{mm}$ main axis). The probes of the sensor were attached on the face using adhesive tape. They were placed parallel to each other and perpendicular to the midline of the face, one in the horizontal dimple just above the chin and the other one on the forehead (Fig. 1). The sensor was connected to the distance-meter electronic module, which converted the distance between the two sensor probes into a voltage. The signal was digitalized with a sampling frequency of $10 \mathrm{~Hz}$, transmitted by cable to the polysomnograph, and could be processed off-line. The signal represents the degree of the mouth opening through the time with a precision of $0.1 \mathrm{~mm}$. Fig. (1) shows the placement of the mandible movement sensor.

\section{c. Manual Scoring}

All the recordings were analyzed between a START time (lights out) and a STOP time (lights on). Sleep stages, arous- als and breathing events were scored according to AASM rules [8]. Apnea and hypopnea index (AHI) and arousal index (ArI) were then computed over the total sleep time (TST).

\section{d. Mandible Movement Processing}

First, for each recording, the zero value of the mandible movement signal was attributed to the fully closed mouth level. The polysomnograph had been set so that, as the distance between the two sensor probes increases, the level of the recorded signal actually decreases (simple signal reversal). Therefore, the more open the mouth gets (the more the mandible lowers), the more negative the value of the signal gets (the more the mandible movement signal lowers). Second, the average value of the mandible movement signal over all the sleep periods (aLOW stands for average lowering) was computed. Third, the average over all the sleep periods of the instantaneous amplitude of the oscillating component of the signal in the breathing frequency band [0.150.33 ] $\mathrm{Hz}$ (aAMPL stands for average amplitude, see [9] for more details about the signal processing) was computed. Thus, while aLOW reflects the mean level of mouth opening during sleep, aAMPL reflects the mean amplitude of the mandible oscillations. Fig. (2) illustrates the computation of these two parameters.

\section{e. Statistical Analysis}

Four parameters (AHI, ArI, aLOW and aAMPL) were computed for each recording. The mean and standard deviation of these parameters were computed within each of the two groups (OSA and CPAP). Statistical difference between groups was assessed under the $\mathrm{R}$ software [10] by reducing the input space dimensionality through principal component analysis followed by the non-parametric Wilcoxon rank-sum test.

\section{RESULTS}

The DPSG and CPSG recordings from thirty-four OSA patients were collected and formed the groups 1 (before CPAP) and 2 (with CPAP). These 34 patients ( 27 men and 7 women) were $52 \pm 9$ year-old and had a BMI of $33.0 \pm 8.0$. The applied CPAP pressure was $9.0 \pm 1.2$.

Table 1 gives the mean and standard deviation of the four computed parameters (AHI, ArI, aLOW, and aAMPL). It shows that the CPAP is effective in reducing the AHI and 
(a) 6-hour recording
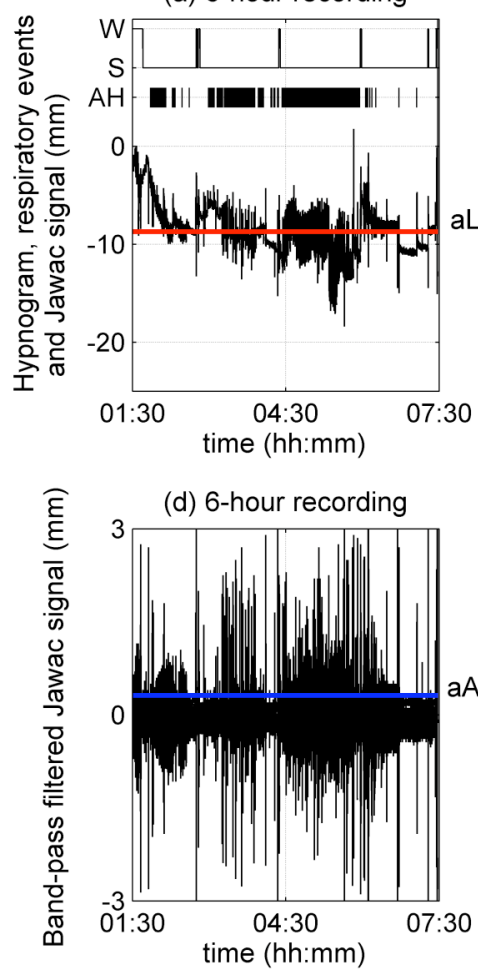

(b) 3-minute normal breathing

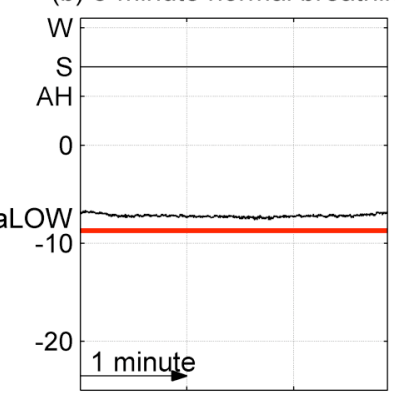

(e) 3-minute normal breathing

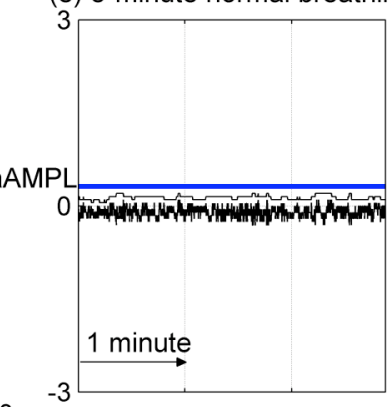

(c) 3-minute abnormal breathing

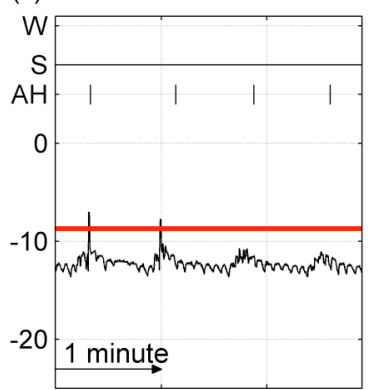

(f) 3-minute abnormal breathing

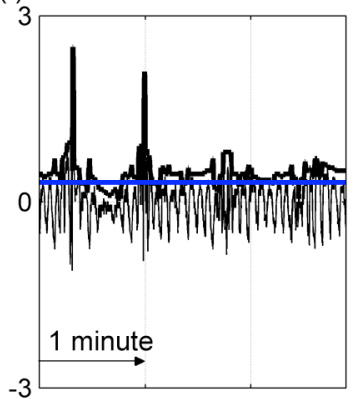

Fig. (2). Illustration of the of aLOW and aAMPL parameters: (a) the mandible movement (Jawac) signal over 6-hour recording, the hypnogram (W-wake, S-sleep) and the respiratory events (AH), the red line is the aLOW, the average of the jawac signal on the sleep periods; (b) a zoom on 90 seconds of the mandible movement signal in (a) showing the time behaviour of normal breathing; (c) 90 seconds of the mandible movement signal in (a) showing the time behaviour when obstructive apneas occur; (d) (e) (f) are the band pass filtering of the Jawac signal and the resulting aAMPL parameter, the average of the amplitude of oscillations on the sleep periods.

Table 1. Mean and Standard Deviation of the Parameters (AHI, ArI, aLOW, and aAMPL) in the 2 Groups

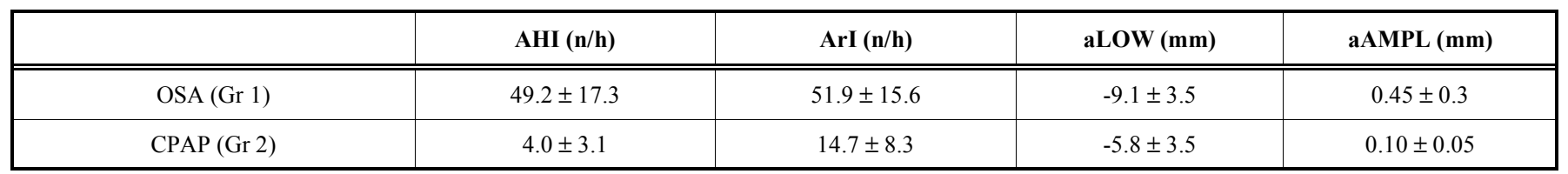

the ArI (mean AHI below 5 and mean ArI below 15 in group 2 ). The average lowering, aLOW, decreased from $-9 \mathrm{~mm}$ in OSA to $-5.8 \mathrm{~mm}$ under CPAP. The average amplitude of the oscillations of the mandible, aAMPL, also drops from $0.45 \mathrm{~mm}$ in OSA down to $0.1 \mathrm{~mm}$ under CPAP. The principal component analysis reduced the dimensionality to one value that explained $96 \%$ if the 4 parameters were considered and $98 \%$ if only the 2 parameters aLOW and aAMPL from mandible movements. The Wilcoxon was then applied and showed a strong difference between the OSA and CPAP groups $(\mathrm{p}<0.0001)$.

Fig. (3) gives the box plots of the 4 parameters for each group. The AHI under CPAP was greater than $10 \mathrm{n} / \mathrm{h}$ in 3 cases while aLOW was in the range $[-5 ;-2]$ and aAMPL in [0.10-0.12]. However, despite the reduction in the mandible movements, respiratory events were still visible in this signal. The aAMPL parameter was $0.4 \mathrm{~mm}$ in one CPAP case but it was not related to any malfunctioning CPAP or leakage, the ideal pressure seemed to be underestimated. The aLOW parameter was increased in four CPAP cases in the range of $[-14 ;-9] \mathrm{mm}$ though the AHI, ArI and aAMPL fell below $7 \mathrm{n} / \mathrm{h}, 10 \mathrm{n} / \mathrm{h}$ and $0.15 \mathrm{~mm}$ respectively. A sensor shifting (rotation) inducing an 8-mm offset was suspected in only one case.

Fig. (4) presents two examples with mandible movement signal, scored apneas/hypopneas $(\mathrm{AH})$ and related hypnogram (W for wake, $\mathrm{S}$ for sleep), one example per group. In these examples the mandible movement behavior is characterized by a permanent activity and a wide open mouth in severe OSA, but this high activity vanishes and the mouth becomes more closed when CPAP pressure is applied.

\section{DISCUSSION}

Several studies showed that the mouth is more open in OSA patients than in healthy subjects, especially during a respiratory event and at the end of expiration [1-4]. Our study showed that in OSA (AHI greater than 25, $n=34$ ), the mandible experiences significant oscillations related to effort $(0.45 \mathrm{~mm}$ average amplitude, aAMPL) and the opening of the mouth is of more than $9 \mathrm{~mm}$ in average (aLOW). This 
(a)

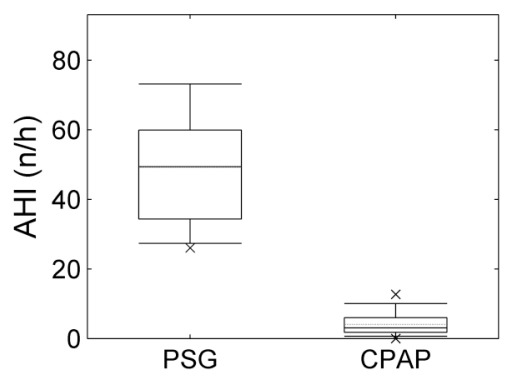

(c)

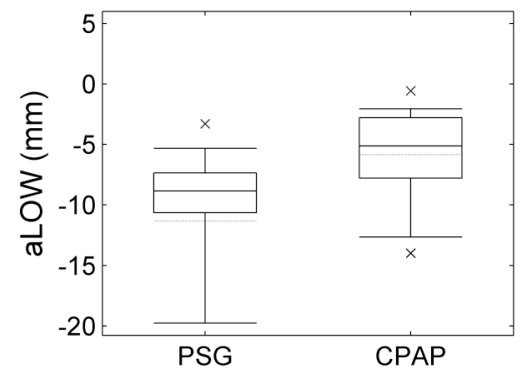

(b)

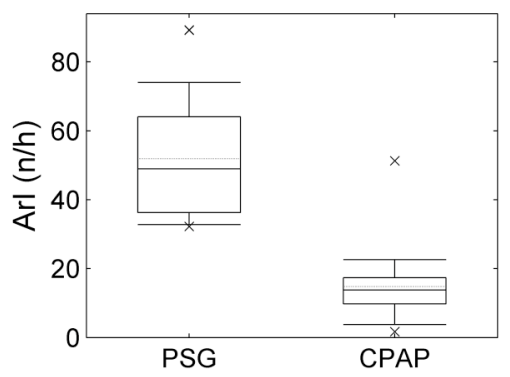

(d)

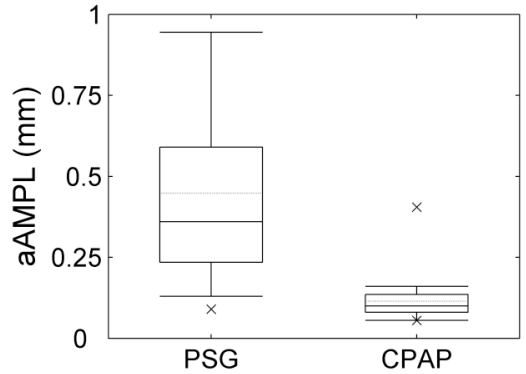

Fig. (3). Box plots of the four parameters AHI (a), ArI (b), aLOW (c), and aAMPL (d). The crosses indicate the extrema, the box height covers the interquartile range (between $25^{\text {th }}$ and $75^{\text {th }}$ percentile), the solid line in each box corresponds to the median and the dashed line is the mean.

Two recordings from one patient: diagnostic PSG and CPAP control PSG recordings
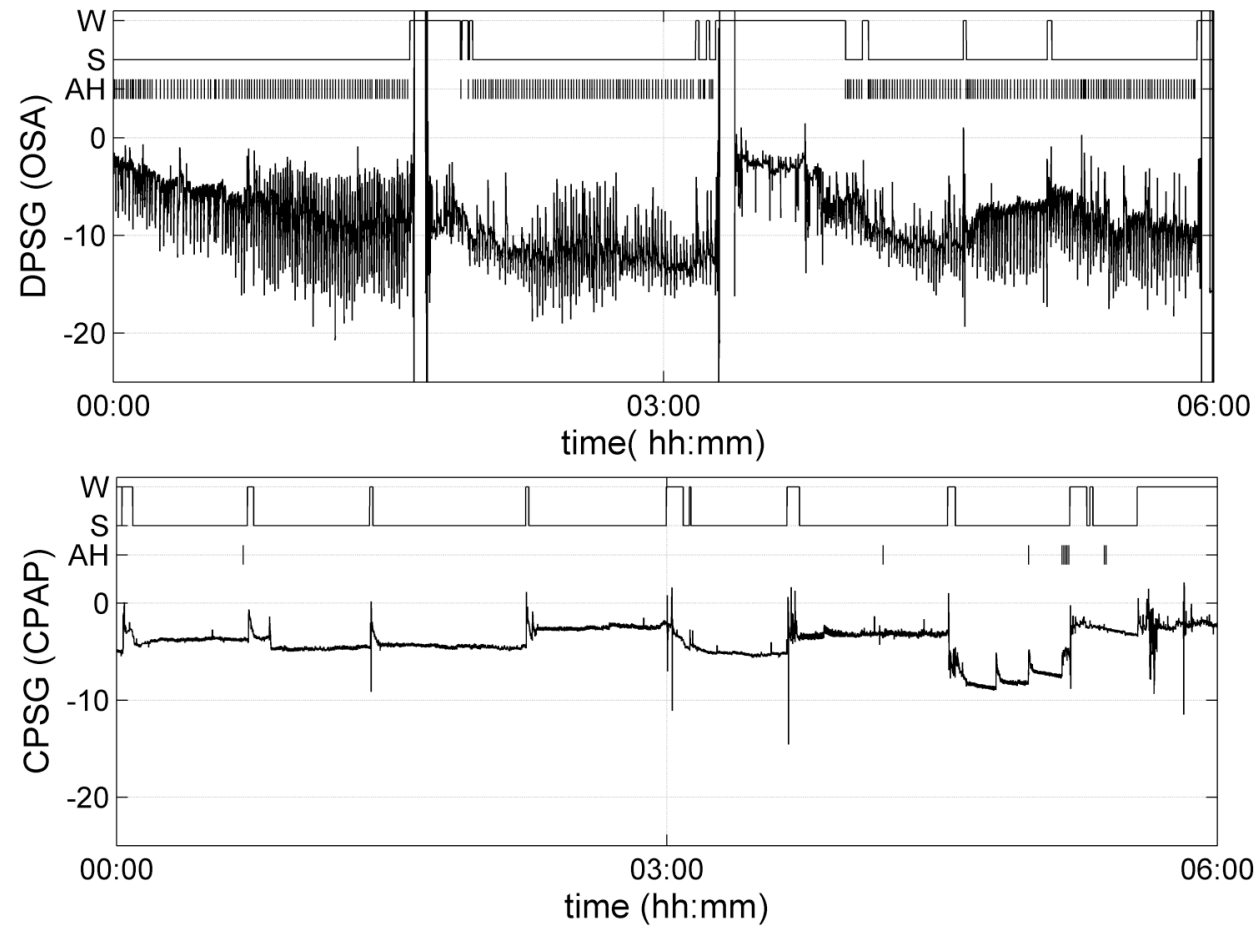

Fig. (4). Two examples, one for each group, of the mandible movement signal (in $\mathrm{mm}$ ), the scored apneas and hypopneas (AH), and the sleep/wake state: (upper graph) OSA diagnostic recording characterized by oscillating mandible movements and a wide open mouth and (lower graph) the corresponding CPAP control recording where the mandible movement signal is much more stable and closer to 0 , thus the mouth is less open.

mandible behavior was very different from the behavior observed in the related CPAP recordings (same 34 patients,
aAMPL $=0.1 \mathrm{~mm}$ and aLOW $=6 \mathrm{~mm}$ with $\mathrm{p}<0.0001)$. These findings corroborated published results: OSA patients are 
characterized by a wider mouth opening. Since mouth opening increases the risk of occlusion, the CPAP treatment reduces the degree of mouth opening and the effort to breathe because it prevents upper airways from collapsing.

No knowledge about nose or mouth breathers (NBs and MBs) was included in this study. Bachour et al., showed that the time spent breathing by the mouth in MBs under nasal CPAP decreased from $84.9 \%$ down to $19.9 \%$ in average after 3 months [11]. As a nasal CPAP mask was used in this study and the inferior jaw was thus free to move, changes observed in the mandible movement signal between DPSG and CPSG recordings were due to passive or natural physiological effects.

Four CPAP cases did not close their mouth under treatment but opened it instead. We suspected a sensor shifting of several millimetres in only one case. In the other three cases, air leakage by the mouth should be speculated but the AHI was below $7 \mathrm{n} / \mathrm{h}$ indicating an efficient treatment. The CPAP pressure was therefore not changed. Another patient did have an important oscillatory component under CPAP (average amplitude around $0.4 \mathrm{~mm}$ ). This latter case could be explained by an underestimation of the ideal pressure though respiratory events were drastically reduced (AHI under $\mathrm{CPAP}=1 \mathrm{n} / \mathrm{h}$ ). In three patients for who the AHI was greater than $10 \mathrm{n} / \mathrm{h}$ under CPAP, the two average mandible parameters (aLOW and aAMPL) were small (aLOW in $[-5 ;-2]$ and aAMPL in [0.10-0.15]). Nevertheless, a fine observation of mandible movement signal allowed to point out most abnormal respiratory events.

This study suffered from its retrospective nature, very restrictive inclusion criteria have been considered to select patients. We did not have full control on how the data have been acquired to ensure that the mandible sensor did not shift for instance which is critical for the computation of the average lowering parameter. Moreover, the comparison should be extended to multiple nights from the same patients to check the stability of the computed parameters. Further studies should focus on efficiency control or even on titration of CPAP and/or of mandibular advancement devices using monitoring of mandible movements. Special attention should be paid to distinguish nasal and mouth breathers and to check if differences exist between them. CPAP titration by mandible movement processing has already been shown feasible and promising in a preliminary study [12].

\section{CONCLUSIONS}

When an efficient CPAP pressure is applied, the mandible behaves much more like in healthy subjects, i.e. a more closed mouth, fewer broad sharp mouth closures, and absence of, or at least very small, oscillating mandible movements. Mandible movement behavior could be a simple and reliable indicator of CPAP effectiveness.

\section{CONFLICT OF INTEREST}

None declared.

\section{ACKNOWLEDGEMNT}

None declared.

\section{REFERENCES}

[1] Lavie P. Rediscovering the importance of nasal breathing in sleep or, shut your mouth and save your sleep. J Laryngol Otol 1987; 101(6): 558-63.

[2] Meurice JC, Marc I, Carrier G, Series F. Effects of mouth opening on upper airway collapsibility in normal sleeping subjects. Am J Respir Crit Care Med 1996; 153(1): 255-9.

[3] Van de Graaf WB. Thoracic influence on upper airway patency. J Appl Physiol 1988; 65: 2124-31.

[4] Hollowel DE, Surrat PM. Mandible position and activation of submental and masseter muscles during sleep. J Appl Physiol 1991; 71(6): 2267-73.

[5] Poirrier R, Chakar B, Lacroix A, Dive D, Hansen I, Frank G. Relationship between mouth opening and intrathoracic pressure during sleep apnea. In Somnologie suppl. of the 5th World Congress on Sleep Apnea 1997; 2: 37.

[6] Miyamoto K, Özbek MM, Lowe AA, et al. Mandibular posture during sleep in patients with obstructive sleep apnoea. Arch Oral Biol 1999; 44: 657-64

[7] Stradling JR, Hardinge M, Paxton J, Smith DM. Relative accuracy of algorithm-based prescription of nasal CPAP in OSA. Respir Med 2004; 98: 152-4.

[8] The Report of an American Academy of Sleep Medicine Task Force. Sleep-related breathing disorders in adults: recommendations for syndrome definition and measurement techniques in clinical research. Sleep 1999; 22: 667-89.

[9] Senny F, Destiné J, Verly J, Ansay P, Poirrier R. Automatic scoring of sleep apnea and hypopnea by the analysis of mandibular movements. In Proceedings of the 3rd IASTED international conference on Biomedical Engineering 2005; 373-8.

[10] R Development Core Team(2011) R: a language and environment for statistical computing, reference index version 2.13.0. R Foundation for Statistical Computing, Vienna. SBN 3-900051-07-0. Available at: http://www.R-project.org. [Accessed: 09 December 2011].

[11] Bachour A, Maasilta P. Mouth breathing compromises adhenrence to nasal continuous positive airway pressure therapy. Chest 2004; 126: $1248-54$

[12] Poirrier R, Cambron L, Moonen G, et al. Disappearance of mouth opening and mouth oscillations : a marker of efficient NCPAP treatment of OSA, Oral presentation at the World Congress on Sleep Apnea (WCSA) 2009, Seoul, Korea. 